\title{
Influência da estação do ano nas características do sêmen e na concentração de hormônios em touros Nelore e Simental
}

\author{
[Influence of year season on semen characteristics and hormonal levels \\ in Nelore and Simental bulls] \\ M.G.M. Chacur ${ }^{1}$, K.T. Mizusaki ${ }^{2}$, F.H. Santos ${ }^{3}$, A.G. Cesare $^{3}$, L.R.A. Gabriel filho ${ }^{4}$, \\ E. $\mathrm{Oba}^{5}$, A.A. Ramos $^{6}$ \\ ${ }^{1}$ Faculdade de Ciências Agrárias - Universidade do Oeste Paulista-UNOESTE - Presidente Prudente, SP \\ ${ }^{2}$ Aluno de pós-graduação - UNOESTE - Presidente Prudente, SP \\ ${ }^{3}$ Médicos Veterinários autônomos \\ ${ }^{4}$ UNESP - Campus Tupã, SP \\ ${ }^{5}$ UNESP-FMVZ, Botucatu, SP \\ ${ }^{6}$ UNESP-FCA, Botucatu, SP

\section{RESUMO}

Estudou-se a influência das quatro estações do ano nas características do sêmen e nas concentrações de testosterona e cortisol em touros. Cinco touros Nelore e cinco Simental entre 48 e 72 meses de idade, criados extensivamente, foram avaliados andrologicamente por meio de exames físicos das características morfológicas do sêmen e das concentrações séricas de testosterona e cortisol. Houve redução na motilidade e no vigor do sêmen no inverno $(\mathrm{P}<0,05)$ na raça Simental. Observou-se correlação $(\mathrm{P}<0,01)$ entre testosterona $\mathrm{x}$ motilidade $(0,69)$ e testosterona $\mathrm{x}$ vigor $(0,57)$ na raça Simental, e cortisol $\mathrm{x}$ motilidade $(0,68)$ e cortisol $x$ vigor $(0,65)$ na raça Nelore. O efeito das estações do ano modificou a qualidade do sêmen com aumento da motilidade e vigor espermáticos na primavera e verão nos touros Simental. A concentração de cortisol diminuiu no outono nos touros Nelore.

Palavras-chave: touro, testosterona, cortisol, sêmen, sazonalidade

\begin{abstract}
The influence of four seasons per year on semen characteristics and levels of testosterone and cortisol in Nelore and Simental bulls was studied. Five Nelore and five Simental bulls extensively managed, at 48-72 months old were evaluated for sexual soundness using physical and morphological characteristics of semen and serum levels of testosterone and cortisol. In Simental bulls there was decreased motility and vigor of semen $(P<0.05)$ during winter. There was a correlation $(P<0.01)$ between testosterone $x$ motility $(0.69)$ and testosterone $x$ vigor (0.57) in the Simental breed and cortisol $x$ motility (0.68) and cortisol $x$ vigor (0.65) in Nelore the breed. The effect of seasons during the year changed the semen quality with increased sperm motility and vigor in spring-summer in Simental bulls. The cortisol level decreased in autumn in Nelore bulls.
\end{abstract}

Keywords: bull, testosterone, cortisol, semen, seasonality

\section{INTRODUÇÃO}

A baixa eficiência reprodutiva em touros pode ser causada por mudanças climáticas que afetam a gametogênese (Vale Filho, 2001). A queda na qualidade do sêmen pode ocorrer em razão do desconforto térmico dos animais frente à elevada temperatura (Galina e Arthur, 1991).

A redução da motilidade espermática progressiva, a queda da produção espermática e a elevação da porcentagem de espermatozoides com alterações morfológicas são causadas

Recebido em 28 de abril de 2011 
pela elevação da temperatura testicular (Kastelic et al., 1996). A interação ambienteespermatogênese resulta na habilidade dos touros para produzir sêmen (Taylor et al., 1985). No Brasil, Koivisto et al. (1998) relataram maior percentual de espermatozoides anormais perante altas temperaturas e umidade relativa do ar, e Silva et al. (2009), em condições ambientais similares, observaram maior volume dos ejaculados nos períodos chuvosos.

A relação entre os fatores do clima com o sêmen foi abordada por Galina e Arthur (1991), em touros nos trópicos, salientando a importância da variação sazonal sobre a concentração espermática e a porcentagem de espermatozoides com alterações morfológicas, indicando que a baixa qualidade do sêmen, em alguns animais, pode ocorrer em razão do desconforto causado por temperaturas ambientes elevadas. Fonseca et al. (1992), ao estudarem a raça Nelore, concluíram que a qualidade do sêmen pode ser afetada significativamente pelo ambiente, por meio de flutuações de temperatura, umidade e fotoperíodo.

Em relação às estações do ano, a qualidade do sêmen foi inferior no verão, resultando na queda da taxa de prenhez em vacas (Barth e Waldner, 2002) e refletindo na eficiência reprodutiva e produtiva, diretamente relacionada à contribuição do touro (Silva et al., 1991). No Brasil, a interferência ambiental nas características físicas e na morfologia espermática de touros tem sido estudada em centrais de inseminação artificial, com animais em regime permanente de colheita de sêmen (Brito et al., 2002; Silva et al., 2003). Observa-se a necessidade da melhoria genética dos rebanhos para identificar reprodutores superiores, resultando no aumento do lucro, sendo esse um ponto relevante para a oferta de touros (Dias et al., 2007).

O eixo hipotálamo-hipófise-adrenal (HPA) controla a resposta neuroendócrina durante $\mathrm{O}$ estado de estresse, elevando a concentração plasmática de cortisol (Charmandari et al., 2005), fundamental para a adaptação do animal ao meio ambiente. Anderson (1992) encontrou correlação positiva entre a concentração de testosterona sérica e a fertilidade de touros, avaliada pela taxa de não retorno das vacas ao estro. Constatou, também, que os animais com maior motilidade espermática apresentaram, em média, maior concentração de testosterona.

Considerando-se a predominância da criação extensiva e a monta natural faz-se necessária a investigação dos fatores climáticos e dos efeitos das estações do ano sobre as características seminais de touros mantidos no campo (Oliveira et al., 2006). Levando-se em consideração as informações publicadas pelos autores supracitados, justifica-se estudar a qualidade seminal e as concentrações de hormônios em touros criados de forma extensiva.

O objetivo do presente estudo foi investigar a influência da estação do ano nas características do sêmen e na concentração de testosterona e cortisol, em touros das raças Nelore e Simental, criados extensivamente.

\section{MATERIAL E MÉTODOS}

Foram utilizados touros das raças Nelore $(n=5)$ e Simental $(n=5)$, com idades entre 48 e 72 meses, criados extensivamente em pasto de Brachiaria decumbens, com sal mineral e água ad libitum. Os animais foram submetidos às avaliações clínicas e espermáticas, para efeito de seleção para monta natural, segundo normas do Colégio Brasileiro de Reprodução Animal (Manual...,1998).

O município de Presidente Prudente-SP, onde o experimento foi realizado, está localizado na latitude $21^{\circ} 29^{\prime} 50^{\prime \prime} \mathrm{S}$; longitude $49^{\circ} 14^{\prime} 20^{\prime \prime} \mathrm{W}$ e altitude de $475 \mathrm{~m}$. Em 2008/2009, os fatores climáticos aferidos para primavera $(\mathrm{P})$, verão (V), outono (O) e inverno (I) foram: temperatura ambiente média $\mathrm{P}-25,9 ; \mathrm{V}-26,6 ; \mathrm{O}-21,9$ e I - 21, $1^{\circ} \mathrm{C}$, insolação ( $\mathrm{P}$ - 400,9; V - 464,0; O 721,3 e I - 142,6 horas), índice pluviométrico cumulativo (P - 291,8; V - 925,0; O - 273,0 e I $-191,8 \mathrm{~mm})$ e umidade relativa média $(\mathrm{P}-65,9$; V - 71,7; O - 70,1 e I - 66,7\%). O clima é caracterizado pela presença de massas de ar tropical e polar, com estação de inverno fria e seca e de verão quente e chuvoso.

Dezesseis colheitas de sêmen por meio de eletroejaculador automático (NEOVET, mod. Autoejac $\left.{ }^{\circledR}\right)$ foram efetuadas em cada um dos 10 touros, perfazendo 160 amostras analisadas. O experimento foi realizado no período de setembro de 2008 a agosto de 2009, sendo 
efetuadas quatro colheitas em cada uma das quatro estações do ano. As alterações morfológicas espermáticas foram classificadas de acordo com Barth e Oko (1989).

No mesmo manejo da colheita de sêmen foram realizadas colheitas de sangue, por venopunção jugular, com oito repetições por touro, duas por estação do ano, totalizando 80 amostras para cada um dos hormônios quantificados, seguida por centrifugação do material a $1500 \mathrm{~g} / 15 \mathrm{~min}$, separando e estocando o soro em criotubos (Eppendorf®) de 1,5mL; identificados e estocados a $-20^{\circ} \mathrm{C}$ até o processamento. As análises para a obtenção da concentração sérica de testosterona $(\mathrm{ng} / \mathrm{dL})$ e cortisol $(\mu \mathrm{g} / \mathrm{dL})$ foram realizadas com kits comercias (DPC-Medlab ${ }^{\circledR}$ ), em fase solida, e as quantificações dos hormônios realizadas por radioimunoensaio (RIA).

Adotou-se o delineamento em blocos ao acaso, em arranjo fatorial $4 \times 2$, composto de quatro estações do ano, primavera, verão, outono e inverno, e duas raças, Simental e Nelore. Os dados relativos às características do sêmen e aos hormônios foram submetidos à análise de variância, e as diferenças entre as médias comparadas pelo teste de Tukey a $5 \%$.

O projeto de pesquisa foi aprovado junto ao Comitê de Ética em Pesquisa da UNOESTE, em 29/10/2008, sob o protocolo 135/08.

\section{RESULTADOS E DISCUSSÃO}

Para o volume do ejaculado na raça Simental, houve diferença $(\mathrm{P}<0,05)$ entre o verão e o inverno, com superioridade da primeira estação para a variável estudada (Tab. 1). Os valores médios do volume dos ejaculados na raça Simental foram semelhantes aos descritos por Igna et al. (2010).

Nas estações do verão e outono, na raça Simental, um maior volume de ejaculado foi encontrado, em relação à Nelore $(\mathrm{P}<0,05)$. O maior volume do ejaculado ocorreu durante a estação do verão, semelhante ao relato de Silva et al. (2009).

Tabela 1. Média e desvio-padrão para as características do sêmen nas quatro estações do ano para touros das raças Simental $(\mathrm{S})$ e Nelore $(\mathrm{N})$, criados extensivamente

\begin{tabular}{llcccc}
\hline Variáveis & Raça & Primavera & Verão & Outono & Inverno \\
\hline Volume & $\mathrm{S}$ & $8,80 \pm 0,65 \mathrm{Aab}$ & $9,85 \pm 0,65 \mathrm{Aa}$ & $8,76 \pm 0,58 \mathrm{Aab}$ & $7,35 \pm 0,65 \mathrm{Ab}$ \\
& $\mathrm{N}$ & $7,10 \pm 0,65 \mathrm{Aa}$ & $7,55 \pm 0,65 \mathrm{Ba}$ & $6,26 \pm 0,58 \mathrm{Ba}$ & $6,05 \pm 0,65 \mathrm{Aa}$ \\
Motilidade & $\mathrm{S}$ & $70.00 \pm 5.83 \mathrm{Aa}$ & $70.00 \pm 5.83 \mathrm{Aa}$ & $60.80 \pm 8.21 \mathrm{Aab}$ & $48.00 \pm 5.83 \mathrm{Ab}$ \\
& $\mathrm{N}$ & $63.50 \pm 5.83 \mathrm{Aa}$ & $60.00 \pm 5.83 \mathrm{Aa}$ & $54.40 \pm 5.21 \mathrm{Aa}$ & $53.50 \pm 5.83 \mathrm{Aa}$ \\
Vigor & $\mathrm{S}$ & $3.35 \pm 0.28 \mathrm{Aa}$ & $3.55 \pm 0.28 \mathrm{Aa}$ & $3.00 \pm 0.25 \mathrm{Aab}$ & $2.20 \pm 0.28 \mathrm{Ab}$ \\
& $\mathrm{N}$ & $3.05 \pm 0.28 \mathrm{Aa}$ & $2.95 \pm 0.28 \mathrm{Aa}$ & $2.32 \pm 0.25 \mathrm{Aa}$ & $2.50 \pm 0.28 \mathrm{Aa}$ \\
Def. maiores & $\mathrm{S}$ & $11,33 \pm 1,31 \mathrm{Aab}$ & $8,00 \pm 1,07 \mathrm{Ab}$ & $10,75 \pm 0,98 \mathrm{Aab}$ & $12,18 \pm 1,20 \mathrm{Aa}$ \\
& $\mathrm{N}$ & $6,30 \pm 1,07 \mathrm{Ba}$ & $6,31 \pm 1,10 \mathrm{Aa}$ & $9,92 \pm 0,96 \mathrm{Aa}$ & $9,42 \pm 1,10 \mathrm{Aa}$ \\
Def. menores & $\mathrm{S}$ & $7,88 \pm 0,83 \mathrm{Aa}$ & $7,25 \pm 0,79 \mathrm{Aa}$ & $8,25 \pm 0,72 \mathrm{Aa}$ & $10,25 \pm 0,88 \mathrm{Aa}$ \\
& $\mathrm{N}$ & $7,60 \pm 0,79 \mathrm{Aa}$ & $5,63 \pm 0,81 \mathrm{Aa}$ & $7,16 \pm 0,70 \mathrm{Aa}$ & $6,84 \pm 0,81 \mathrm{Ba}$ \\
Def. totais & $\mathrm{S}$ & $19,22 \pm 1,63 \mathrm{Aab}$ & $15,25 \pm 1,54 \mathrm{Ab}$ & $19,00 \pm 1,41 \mathrm{Aab}$ & $22,43 \pm 1,73 \mathrm{Aa}$ \\
& $\mathrm{N}$ & $13,90 \pm 1,53 \mathrm{Ba}$ & $11,97 \pm 1,59 \mathrm{Aa}$ & $17,08 \pm 1,38 \mathrm{Aa}$ & $16,36 \pm 1,59 \mathrm{Ba}$ \\
Concentração & $\mathrm{S}$ & $1,35 \pm 0,13 \mathrm{Aa}$ & $1,38 \pm 0,13 \mathrm{Aa}$ & $1,32 \pm 0,12 \mathrm{Aa}$ & $1,00 \pm 0,13 \mathrm{Aa}$ \\
& $\mathrm{N}$ & $0,88 \pm 0,13 \mathrm{Ba}$ & $1,14 \pm 0,13 \mathrm{Aa}$ & $0,95 \pm 0,12 \mathrm{Ba}$ & $0,91 \pm 0,13 \mathrm{Aa}$ \\
\hline
\end{tabular}

Nível de significância de 5\% (P<0,05); A, B - letras maiúsculas distintas, nas colunas, diferem entre si $(\mathrm{P}<0,05) ; \mathrm{a}, \mathrm{b}$ - letras minúsculas distintas, na linha, diferem entre si $(\mathrm{P}<0,05)$. Volume $-\mathrm{mL}$, motilidade espermática - $\%$, vigor espermático - 1 a 5 , concentração espermática $-\times 10 \% / \mathrm{mL}$, defeitos maiores - \% (def. maiores), defeitos menores - \% (def. menores), defeitos totais - \% (def. totais). 
Para a raça Simental, não houve queda na qualidade do sêmen no verão, com temperatura média de $26,6^{\circ} \mathrm{C}$, em relação às demais estações do ano, conforme as variáveis estudadas (Tab. 1). A despeito de os touros Simental viverem na mesma área geográfica da propriedade rural, desde o nascimento, associada à pequena oscilação da temperatura ambiente média nas estações do ano, durante os 12 meses do experimento, verificou-se diferença significativa $(\mathrm{P}<0,05)$ para a motilidade espermática entre a estação do verão, a qual foi maior em relação à do inverno (Tab.1). Na Romênia, em touros da raça Simental, a qualidade do sêmen foi afetada quando em temperatura ambiente acima de $20^{\circ} \mathrm{C}$ (Igna et al., 2010).Não houve diferença significativa $(\mathrm{P}>0,05)$ entre raças para motilidade e vigor espermáticos (Tab. 1). No inverno, essas características do sêmen apresentaram queda na raça Simental, em relação à primavera e ao verão $(\mathrm{P}<0,05)$. Possivelmente, a maior exigência nutricional da raça Simental interferiu nessas características, especialmente no período das secas, em que a qualidade da pastagem fica prejudicada; diferença essa não observada para a motilidade e vigor $(\mathrm{P}>0,05)$ na raça Nelore para as estações do ano, semelhante ao descrito por Chacur et al. (2011) para a motilidade e diferindo dos relatos de Oliveira et al. (2006) com aumento da motilidade espermática em Nelores na estação chuvosa durante o verão.

No inverno, para a raça Nelore, os valores para motilidade e vigor foram similares aos de Dias et al. (2007), obtidos no Mato Grosso do Sul, na mesma estação climática, provavelmente devido à proximidade geográfica dos locais dos experimentos.
Com relação à morfologia espermática para os defeitos maiores e totais, houve diferença $(\mathrm{P}<0,05)$ entre raças na primavera, com maiores porcentagens observadas na raça Simental. No inverno, para os defeitos menores e totais, houve diferença entre raças $(\mathrm{P}<0,05)$, sendo a porcentagem de defeitos superiores para $\mathrm{o}$ Simental.

Na raça Simental, houve diferença $(\mathrm{P}<0,05)$ entre as estações do verão e inverno, para os defeitos espermáticos maiores e totais, com melhor qualidade morfológica dos espermatozoides no verão. Possivelmente, o incremento do aporte nutricional na estação do verão possa ter contribuído de forma positiva na espermatogênese, processo esse dependente da síntese de testosterona. $\mathrm{Na}$ raça Nelore, não houve diferença $(\mathrm{P}>0,05)$ entre as estações do ano para a morfologia espermática, semelhante ao relato de Chacur et al. (2011) para os defeitos espermáticos maiores na mesma estação do ano e diferindo dos descritos por Oliveira et al. (2006), nos quais em touros Nelore, obteve-se menor percentual de defeitos maiores na estação do verão, denominada como chuvosa.

A concentração espermática, entre raças, na primavera e no outono, revelou diferença $(\mathrm{P}<0,05)$, a qual foi superior para a Simental. Dentro das raças, não houve diferença $(\mathrm{P}>0,05)$ para a concentração espermática nas quatro estações do ano.

Vale salientar que o método de colheita por eletroejaculação pode influenciar no volume de sêmen obtido, fator esse minimizado no presente estudo pelo uso do aparelho eletrônico para eletroejaculação, o qual funciona com impulsos elétricos padronizados durante as colheitas.

Tabela 2. Média e desvio-padrão para as concentrações de testosterona (ng/dL) e cortisol ( $\mu \mathrm{g} / \mathrm{dL})$ nas quatro estações do ano para touros das raças Simental $(\mathrm{S})$ e Nelore $(\mathrm{N})$, criados extensivamente

\begin{tabular}{llcccc}
\hline Variáveis & Raça & Primavera & Verão & Outono & Inverno \\
\hline \multirow{2}{*}{ Testosterona } & $\mathrm{S}$ & $879,47 \pm 164,70 \mathrm{Aa}$ & $901,09 \pm 173,61 \mathrm{Aa}$ & $584,02 \pm 164,70 \mathrm{Aa}$ & $648,15 \pm 164,70 \mathrm{Aa}$ \\
& $\mathrm{N}$ & $430,40 \pm 173,61 \mathrm{Aa}$ & $234,71 \pm 196,86 \mathrm{Ba}$ & $420,31 \pm 164,70 \mathrm{Aa}$ & $329,14 \pm 164,70 \mathrm{Aa}$ \\
\multirow{2}{*}{ Cortisol } & $\mathrm{S}$ & $0,62 \pm 0,26 \mathrm{Ba}$ & $0,61 \pm 0,28 \mathrm{Ba}$ & $0,54 \pm 0,26 \mathrm{Ba}$ & $1,09 \pm 0,26 \mathrm{Ba}$ \\
& $\mathrm{N}$ & $1,68 \pm 0,28 \mathrm{Aa}$ & $3,10 \pm 0,31 \mathrm{Aa}$ & $1,35 \pm 0,26 \mathrm{Ab}$ & $3,06 \pm 0,26 \mathrm{Aa}$ \\
\hline
\end{tabular}

Nível de significância de 5\% (P<0,05); A, B - letras maiúsculas distintas, nas colunas, diferem entre si $(\mathrm{P}<0,05) ; \mathrm{a}, \mathrm{b}$

- letras minúsculas distintas, na linha, diferem entre si $(\mathrm{P}<0,05)$. 
Houve diferença $(\mathrm{P}<0,05)$ entre raças para a testosterona, na estação do verão, com concentrações de $901,09 \pm 173,61 \mathrm{ng} / \mathrm{dL}$ para os touros Simental e $234,71 \pm 196,86 \mathrm{ng} / \mathrm{dL}$ para os Nelores (Tab.2). As concentrações foram semelhantes às descritas por Chacur et al. (2007) em pesquisa realizada no período de outubro a dezembro, estação da primavera, relatando diferença $(\mathrm{P}<0,05)$ entre a raça Pardo-Suíça $(116,60 \pm 39,03 n g / d L)$ e Brangus $(230,92 \pm 27,60 \mathrm{ng} / \mathrm{dL})$ para os valores de testosterona.

No verão, para a raça Simental, não houve diferença $(\mathrm{P}>0,05)$ para a concentração de testosterona em relação às demais estações do ano (Tab. 2); resultado esse que difere de Jimenez-Severiano et al. (2003) em touros Simental, relatando no inverno média de 50ng/dL e no outono $70 \mathrm{ng} / \mathrm{dL}$, com diferença $(\mathrm{P}<0,05)$ em relação ao verão e à primavera, com média de 130ng/dL. Possivelmente, as pequenas amplitudes de variação entre as temperaturas médias nas estações do ano, observadas no presente estudo, possam ter colaborado para a não diferença significativa entre essas estações para as concentrações de testosterona.

As concentrações de cortisol, entre raças, foram superiores nos touros Nelore, independente da estação do ano (Tab. 2). Valores semelhantes foram obtidos por Chacur et al.

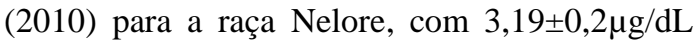
no período de agosto a outubro (invernoprimavera). Com relação à raça Simental, na mesma região geográfica, resultados similares foram descritos por Chacur et al. (2007) em animais Pardo-Suiço e Brangus, com concentrações de $0,31 \pm 0,08 \mu \mathrm{g} / \mathrm{dL}$ e $0,61 \pm 27,60 \mu \mathrm{g} / \mathrm{dL}$, respectivamente.

Para a raça Nelore, houve redução $(\mathrm{P}<0,05)$ nas concentrações de cortisol durante o outono, em relação às demais estações. Supostamente, a temperatura amena dessa estação possa ter influenciado na diminuição da produção do cortisol, devido ao menor estresse.

Tabela 3. Correlações entre testosterona (ng/dL) e as características do sêmen nas quatro estações do ano para touros das raças Simental $(\mathrm{S})$ e Nelore $(\mathrm{N})$, criados extensivamente

\begin{tabular}{|c|c|c|c|c|c|c|}
\hline Variáveis & Raça & Primavera & Verão & Outono & Inverno & Correlações \\
\hline Testosterona & $\mathrm{S}$ & 879,5 & 901,1 & 584,0 & 648,2 & \\
\hline Volume & $S$ & 8,800 & 9,444 & 10,800 & 7,500 & $-0,16$ \\
\hline Cor & $S$ & 1,700 & 2,111 & 1,400 & 1,200 & 0,86 \\
\hline Aspecto & S & 1,800 & 1,889 & 1,400 & 1,900 & 0,62 \\
\hline Turbilhão & $\mathrm{S}$ & 1,800 & 4,000 & 2,800 & 1,800 & 0,31 \\
\hline Motilidade & S & 67,000 & 80,000 & 64,000 & 46,000 & 0,69 \\
\hline Vigor & S & 3,000 & 4,111 & 3,200 & 2,100 & 0,57 \\
\hline Concentração & S & 1,093 & 1,697 & 1,360 & 0,960 & 0,37 \\
\hline Def. maiores & S & 13,889 & 6,222 & 10,800 & 10,875 & $-0,19$ \\
\hline Def. menores & $\mathrm{S}$ & 7,889 & 6,222 & 7,400 & 8,250 & $-0,47$ \\
\hline Def. totais & $\mathrm{S}$ & 21,778 & 12,444 & 18,200 & 19,125 & $-0,26$ \\
\hline Testosterona & $\mathrm{N}$ & 430,41 & 234,71 & 420,31 & 329,15 & correlações \\
\hline Volume & $\mathrm{N}$ & 8,278 & 8,286 & 5,700 & 5,800 & $-0,28$ \\
\hline Cor & $\mathrm{N}$ & 1,444 & 3,000 & 1,600 & 2,200 & $-1,00$ \\
\hline Aspecto & $\mathrm{N}$ & 1,889 & 2,429 & 1,500 & 2,000 & $-0,89$ \\
\hline Turbilhão & $\mathrm{N}$ & 2,222 & 3,714 & 1,300 & 2,200 & $-0,87$ \\
\hline Motilidade & $\mathrm{N}$ & 63,333 & 80,000 & 33,000 & 56,000 & $-0,71$ \\
\hline Vigor & $\mathrm{N}$ & 3,111 & 4,000 & 1,500 & 2,600 & $-0,70$ \\
\hline Concentração & $\mathrm{N}$ & 0,913 & 1,329 & 0,701 & 1,080 & $-0,93$ \\
\hline Def. maiores & $\mathrm{N}$ & 5,889 & 5,857 & 10,700 & 9,200 & 0,36 \\
\hline Def. menores & $\mathrm{N}$ & 7,000 & 4,571 & 8,000 & 7,500 & 0,82 \\
\hline Def. totais & $\mathrm{N}$ & 12,889 & 10,429 & 18,700 & 16,900 & 0,56 \\
\hline
\end{tabular}

Volume - mL, cor: 1 - leitoso, 2 - branco leitoso e 3 - branco marmóreo; aspecto: 1 - aquoso, 2 - viscoso e 3 cremoso; turbilhão - 1 a 5 , motilidade espermática - \%, vigor espermático - 1 a 5 , concentração espermática $\mathrm{x} 10^{9} / \mathrm{mL}$, defeitos maiores - \% (def. maiores), defeitos menores - \% (def. menores), defeitos totais - \% (def. totais). 
Na raça Simental, houve correlações entre a testosterona e as características do sêmen, sendo turbilhão $(0,31)$, concentração $(0,37)$ e defeitos totais $(-0,26)$, resultados esses semelhantes aos de Anderson (1992), que obteve correlação entre a concentração sérica de testosterona e a qualidade seminal dos touros.

Para os defeitos espermáticos maiores e totais, no Simental, correlações foram obtidas para a testosterona. Vale salientar que a porcentagem de defeitos espermáticos menores, maiores e totais, independentemente da estação do ano, para as duas raças, não excedeu a preconizada para a aprovação do uso dos machos para a atividade reprodutiva, segundo normas do Manual...(1998). Destaca-se que, para os touros da raça Nelore, quanto maior a concentração de testosterona, uma redução na qualidade do sêmen foi observada. Para os animais da raça Simental, quanto maior a concentração de testosterona, melhor foi a qualidade do sêmen (Tab. 3).

Tabela 4. Correlações entre cortisol $(\mu \mathrm{g} / \mathrm{dL})$ e as características do sêmen nas quatro estações do ano para touros das raças Simental $(\mathrm{S})$ e Nelore $(\mathrm{N})$, criados extensivamente

\begin{tabular}{|c|c|c|c|c|c|c|}
\hline Variáveis & Raça & Primavera & Verão & Outono & Inverno & Correlações \\
\hline Cortisol & $S^{3}$ & 0,6 & 0,6 & 0,5 & 1,1 & \\
\hline Volume & $S$ & 8,800 & 9,444 & 10,800 & 7,500 & $-0,87$ \\
\hline Cor & S & 1,700 & 2,111 & 1,400 & 1,200 & $-0,60$ \\
\hline Aspecto & S & 1,800 & 1,889 & 1,400 & 1,900 & 0,55 \\
\hline Turbilhão & S & 1,800 & 4,000 & 2,800 & 1,800 & $-0,52$ \\
\hline Motilidade & S & 67,000 & 80,000 & 64,000 & 46,000 & $-0,83$ \\
\hline Vigor & S & 3,000 & 4,111 & 3,200 & 2,100 & $-0,79$ \\
\hline Concentração & S & 1,093 & 1,697 & 1,360 & 0,960 & $-0,66$ \\
\hline Def. maiores & $\mathrm{S}$ & 13,889 & 6,222 & 10,800 & 10,875 & 0,10 \\
\hline Def. menores & S & 7,889 & 6,222 & 7,400 & 8,250 & 0,60 \\
\hline Def. totais & $\mathrm{S}$ & 21,778 & 12,444 & 18,200 & 19,125 & 0,22 \\
\hline Cortisol & $\mathrm{N}$ & 1,68 & 3,10 & 1,36 & 3,06 & correlações \\
\hline Volume & $\mathrm{N}$ & 8,278 & 8,286 & 5,700 & 5,800 & 0,14 \\
\hline Cor & $\mathrm{N}$ & 1,444 & 3,000 & 1,600 & 2,200 & 0,87 \\
\hline Aspecto & $\mathrm{N}$ & 1,889 & 2,429 & 1,500 & 2,000 & 0,85 \\
\hline Turbilhão & $\mathrm{N}$ & 2,222 & 3,714 & 1,300 & 2,200 & 0,75 \\
\hline Motilidade & $\mathrm{N}$ & 63,333 & 80,000 & 33,000 & 56,000 & 0,75 \\
\hline Vigor & $\mathrm{N}$ & 3,111 & 4,000 & 1,500 & 2,600 & 0,65 \\
\hline Concentração & $\mathrm{N}$ & 0,913 & 1,329 & 0,701 & 1,080 & 0,91 \\
\hline Def. maiores & $\mathrm{N}$ & 5,889 & 5,857 & 10,700 & 9,200 & $-0,31$ \\
\hline Def. menores & $\mathrm{N}$ & 7,000 & 4,571 & 8,000 & 7,500 & $-0,60$ \\
\hline Def. totais & $\mathrm{N}$ & 12,889 & 10,429 & 18,700 & 16,900 & $-0,43$ \\
\hline
\end{tabular}

Volume - mL, cor: 1 - leitoso, 2 - branco leitoso e 3 - branco marmóreo; aspecto: 1 - aquoso, 2 - viscoso e 3 cremoso; turbilhão - 1 a 5 , motilidade espermática - \%, vigor espermático -1 a 5 , concentração espermática $\mathrm{x} 10 \% \mathrm{~mL}$, defeitos maiores - \% (def. maiores), defeitos menores - \% (def. menores), defeitos totais - \% (def. totais).

Para os touros da raça Simental, houve correlação de 0,22 entre as concentrações de cortisol $\mathrm{x}$ defeitos espermáticos totais e de 0,60 para cortisol $\mathrm{x}$ defeitos espermáticos menores (Tab. 4).

\section{CONCLUSÕES}

Os touros das raças Nelore e Simental produziram boa qualidade de sêmen ao longo das estações do ano. Para a raça Simental, o efeito das estações da primavera e do verão melhora a motilidade e o vigor, e o do inverno revelou efeito contrário nessas características. Na raça Simental, o efeito do inverno em relação ao verão afetou em maior escala a morfologia espermática. A qualidade do sêmen revelou alta correlação positiva com a testosterona. Para a raça Nelore, não houve variações significativas das características do sêmen ao longo das estações do ano, sendo o estresse menor no outono.

\section{AGRADECIMENTOS}

Á UNOESTE, pelo apoio logístico. 


\section{REFERÊNCIAS}

ANDERSSON, M. Relationship between GnRHinduced sperm motility and fertility in Ayrshire bulls. Anim. Reprod. Sci., v.27, p.107-111, 1992.

BARTH, A.D.; OKO, R.J. Abnormal morphology of bovine spermatozoa. 1.ed Ames: Iowa State University, 1989. 285p.

BARTH, A.D.; WALDNER, C.L. Factors affecting breeding soundness classification of beef bulls examined at the Western College of Veterinary Medicine. Can. Vet. J., v.3, p.274-284, 2002.

BRITO, L.C.F.; SILVA, A.E.D.F.; RODRIGUES, L.H. et al. Effects of environmental factors, age and genotype on sperm production and semen quality in Bos indicus and Bos taurus AI bulls in Brazil. Anim. Reprod. Sci., v.70, p.181-190, 2002.

CHACUR, M.G.M.; AURÉLIO, P.T.F.; OBA, E. et al. Influência de um nutracêutico no sêmen, testosterona, cortisol, eritrogama e peso corpóreo em touros jovens Bos taurus indicus. Semina, v.31, p.439$450,2010$.

CHACUR, M.G.M.; CASTILLO, A.M.N.; GUABERTO, L.M. Influence of the dry and rainy seasons upon plasma seminal (SDS-PAGE) and characteristics of the ejaculate from bulls Bos Taurus indicus. Semina, v.32, p.1565-1574, 2011

CHACUR, M.G.M.; SIRCHIA, F.P.; ZERBINATTI, E.P. et al. Relação entre circunferência escrotal, libido, hormônios e características do sêmen em touros Brangus e Pardo-Suiço. Acta Sci. Vet., v.35, p.173179, 2007.

CHARMANDARI, E.; TSIGOS, C.; CHOUSOS, G. Endocrinology of the stress response. Annu. Rev. Physiol., v.67, p.259-284, 2005.

DIAS, J.C.; ANDRADE, V.J.; VALE FILHO, V.R. et al. Caracterização andrológica de touros Nelore criados extensivamente em Mato Grosso do Sul, Brasil. Vet. Not., v.13, p.39-46, 2007.

FONSECA, V.O.; CRUDELI, G.A.; COSTA E SILVA, E.V. et al. Aptidão reprodutiva de touros da raça Nelore: efeito de diferentes estações do ano sobre as características seminais, circunferência escrotal e fertilidade. Arq. Bras. Med. Vet. Zootec., v.44, p.7-15, 1992.

GALINA, C.S.; ARTHUR, G.H. Review of cattle reproduction in the tropics. Part 6. The Male. Anim. Breed. Abst., v.59, p.403-412, 1991.

IGNA,V.; MOJE, A.; MIRCU, C. et al. The influence of some environmental factors and age on semen production of Fleckvieh bulls. Lucrari Stiintifice Med. Vet., v.43, 2010.
JIMENEZ-SEVERIANO, H.; QUINTAL-FRANCO, J.; VEJA-MURILLO, V. et al. Season of the year influences testosterone secretion in bulls administered luteinizing hormone1. J. Anim. Sci., v.81, p.10231029, 2003

KASTELIC, J.P.; COOK, R.B.; COULTER, G.H. et al. Insulating the scrotal neck affects semen quality and scrotal/testicular temperatures in the Bull. Theriogenology, v.45, p.935-942,1996.

KOIVISTO, M.B.; NOGUEIRA, G.P.; COSTA, M.T.A. Seasonal variations of morphological abnormalities in bovine spermatozoa. In: SEMINAR ON ANIMAL REPRODUCTION AND BIOTECHNOLOGY FOR LATIN AMERICA, 4, 1998. Belém. Proceedings... Belém Uppsala: Swedish University of Agricultural Sciences. 1998, v.2, p.5056.

MANUAL para exame andrológico e avaliação de sêmen animal. 2.ed. Belo Horizonte: Colégio Brasileiro de Reprodução Animal, 1998. 49p.

OLIVEIRA, K.M.; DUARTE, A.M.; NASCIMENTO, M.R.B.M. et al. Influência das estações seca e chuvosa sobre as características seminais de touros das raças Nelore, Gir e Holandês criados a pasto. Vet. Not., v.12, p.145-151, 2006.

SILVA, A.E.D.F.; DODE, M.A.; PORTO, J.A. et al. Estacionalidade na atividade sexual de machos bovinos Nelore e mestiços Fleckvieh e Chianina X Nelore: Características biométricas testiculares. Pesq. Agropec. Bras., v.26, p.1745-1750, 1991.

SILVA, A.R.; FERRAUDO, A.S.; PERECIN, D. et al. Efeito da idade do touro e do período de colheita de sêmen sobre as características físicas e morfológicas do sêmen de bovinos de raças europeias e zebuínas. Rev. Bras. Zootec., v.38, p.1218-1222, 2009.

SILVA, A.R.; FERRAUDO, A.S.; RODRIGUES, L.H. et al. Efeito da idade e do período de colheita de sêmen sobre as características físicas e morfológicas do sêmen de bovinos europeus e zebus. In: ZOOTEC AMBIÊNCIA- EFICIÊNCIA E QUALIDADE NA PRODUÇÃO ANIMAL, 13, 2003, Uberaba. Anais... Uberaba: 2003. p.56-61.

TAYLOR, J.F.; BEAN, B.; MARSHALL, C.E. et al. Genetic and environmental components of semen production traits of artificial insemination Holstein bulls. J. Dairy Sci., v.68, p.2703-2722, 1985.

VALE FILHO, V.R. Subfertilidade em touros: parâmetros para avaliação andrológica e conceituação geral. Cad. Tec. Vet. Zootec., n.35, p.81-87, 2001. 